\title{
Magnetic resonance features of meningeal solitary fibrous tumors
}

\author{
$\mathrm{XI} \mathrm{LI}^{1}$, LILIAN TAN ${ }^{1}$, XIAOMING OUYANG ${ }^{2}$, JINDAI JIANG ${ }^{1}$, SUMENG HUANG $^{1}$, \\ YONG HUANG ${ }^{1}$, SHUXIN LI $^{1}$ and DEJI CHEN ${ }^{1}$ \\ Departments of ${ }^{1}$ Radiology and ${ }^{2}$ Pathology, The Second Affiliated Hospital of Guangzhou Medical University, \\ Guangzhou, Guangdong 510260, P.R. China
}

Received May 6, 2016; Accepted June 29, 2017

DOI: $10.3892 / \mathrm{ol} .2018 .8426$

\begin{abstract}
The aim of the present study was to investigate magnetic resonance (MR) features of meningeal solitary fibrous tumors (SFTs) in order to improve their recognition. The study retrospectively analyzed MR manifestations in 12 cases of meningeal SFTs confirmed by surgery. The lesions were analyzed in terms of the site of their growth, growth mode, morphology, size, changes in the MR signal and the edge of the focus. The tumors were malignant in 3 cases, benign in 7 cases and borderline in 2 cases. A total of 3 cases spanned the tentorium cerebelli, 3 spanned the falx and another case was close to the falx. Overall, 4 superficial tumors were accompanied by adjacent bony destruction. One case was located at the triangular area created by the right lateral ventricles. The tumors were all lobular, with a large diameter and more cystic areas in the center of the focus. The signals were often heterogeneous. The parenchyma in the tumors was significantly enhanced. For certain tumors, the signal was usually inhomogeneous and not uniform. The MR features of a SFT were often similar to other intracranial tumors and easily misdiagnosed. There were certain innate characteristics, for example, the tumor was often a solitary large soft-tissue mass with an irregular edge and a clear boundary, with a lobulated contour, and was widely involved with other tissues. The tumors often grew across either the falx or tentorium cerebelli. A significantly inhomogeneous signal was found and either a low or low-high mixed signal on T2-weighted images in particular. These features are useful for the differential diagnosis of SFTs and other tumors.
\end{abstract}

\section{Introduction}

The solitary fibrous tumor (SFT) is a rare type of spindled cell tumor of the mesenchymal tissue (1) whose identity was

Correspondence to: Professor Deji Chen, Department of Radiology, The Second Affiliated Hospital of Guangzhou Medical University, 250 Chang Gang Road, Haizhu, Guangzhou, Guangdong 510260, P.R. China

E-mail: dejichen@126.com

Key words: solitary fibrous tumor, meningeal, magnetic resonance imaging first proposed by Wagner in 1870 (2). In 1931, Klemperer and Coleman (3) first reported their pathological characteristics (4). The tumors often originate in the pleural space $(5,6)$. Although they can occur anywhere in the body, SFTs of the central nervous system (CNS) are rare (7), with Carneiro et al (8) first reporting them in 1996. Due to the distinct pathological type, the incidence of this disease is low and forming a preoperative diagnosis is difficult (9). Furthermore, an SFT is often misdiagnosed as another type of tumor (10), such as a fibrous meningioma. The features of meningeal SFTs are similar to those of other tumors in the CNS in certain respects, such as upon reviews of the pathology or from the imaging perspective. Globally, there are currently few studies on meningeal SFT characteristics in magnetic resonance imaging (MRI) $(11,12)$. At present, there are also few cases reported (13) domestically in China. In order to improve the recognition of the tumors for the diagnosis of an SFT, the data from 12 cases of meningeal SFTs that were treated in the Second Affiliated Hospital of Guangzhou Medical University (Guangzhou, China) and whose diagnosis was confirmed by surgery and pathology, were collected between January 2006 and January 2016. The MRI signs of the SFTs were observed and analyzed. The present study aimed to determine valuable features for a differential diagnosis to separate an SFT from other tumors using preoperative MRI.

\section{Materials and methods}

Case data. In total, 12 cases of meningeal SFTs with complete clinical and MRI data were reviewed in the present study. All patients were treated in the Neurosurgery Unit of the Second Affiliated Hospital of Guangzhou Medical University between January 2006 and January 2016. The inclusion criterion used was that patients had undergone partial or complete surgical resection and were confirmed by pathology as having meningeal SFTs. According to the 2007 World Health Organization (WHO) classification of central nervous system tumors, ISFT was classified as meningeal mesenchymal tumor (WHO Grade 1) (Table I) (14). The study cohort consisted of 6 men and 6 women aged between 20 and 71 years, with a mean age of 48.8 years. This study was conducted in accordance with the declaration of Helsinki and with approval from the Ethics Committee of Guangzhou Medical University. Written informed consent for publication of this study was obtained from the patient or their guardian/family members. 
Table I. Case data.

MRI

Case no. Sex/age, years

Location and MRI findings

Size, $\mathrm{mm}^{\mathrm{a}}$ preoperative diagnosis

\begin{tabular}{|c|c|c|c|c|}
\hline 1 & Male/67 & $\begin{array}{l}\text { Left anterior cranial fossa, across the falx, the deep lobulation, } \\
\text { more cystic areas, inhomogeneous signal, mild edema }\end{array}$ & $60 \times 75 \times 62$ & Meningioma \\
\hline 2 & Male/59 & $\begin{array}{l}\text { Left parietal, with adjacent bony destruction, certain cystic } \\
\text { areas, inhomogeneous signal, no edema }\end{array}$ & $55 \times 53 \times 59$ & Meningioma \\
\hline 3 & Male/53 & $\begin{array}{l}\text { Right tentorium cerebelli, across the tentorium cerebelli, } \\
\text { inhomogeneous signal, mild edema }\end{array}$ & $45 \times 44 \times 48$ & Meningioma \\
\hline 4 & Male/36 & $\begin{array}{l}\text { Right temporal-parietal-occipital junction, with adjacent } \\
\text { bony destruction, certain patches of cystic areas, } \\
\text { inhomogeneous signal, moderate edema }\end{array}$ & $63 \times 64 \times 67$ & Gliobastona \\
\hline 5 & Female/52 & $\begin{array}{l}\text { Left occipital and left cerebellum, across the tentorium } \\
\text { cerebelli, more cystic area, inhomogeneous signal, no edema }\end{array}$ & $44 \times 60 \times 62$ & $\begin{array}{l}\text { Identify meningioma } \\
\text { and SFT }\end{array}$ \\
\hline 6 & Female/40 & $\begin{array}{l}\text { Frontal on each side of the cerebral falx, across the } \\
\text { falx, more cystic areas, inhomogeneous signal, mild edema }\end{array}$ & $71 \times 60 \times 43$ & Meningioma \\
\hline 7 & Female/20 & $\begin{array}{l}\text { Right parietal areas beside sagittal sinus-the falx, deep } \\
\text { lobulation, inhomogeneous signal, with adjacent bony } \\
\text { destruction, no edema }\end{array}$ & $36 \times 33 \times 33$ & Meningioma \\
\hline 8 & Male/50 & $\begin{array}{l}\text { Frontal on each side of the cerebral falx, across the falx, deep } \\
\text { lobulation, more cystic areas, inhomogeneous signal, close to } \\
\text { the adjacent dura, with adjacent bony destruction, moderate } \\
\text { edema, numerous small vesicles }\end{array}$ & $77 \times 55 \times 50$ & Meningioma \\
\hline 9 & Female/45 & $\begin{array}{l}\text { Across the right tentorium cerebelli, at the occipital and right } \\
\text { cerebellum, shallow lobe, few cystic areas, mild } \\
\text { inhomogeneous signal, mild edema, low signal on } \\
\text { T2-weighted imaging }\end{array}$ & $53 \times 45 \times 42$ & Meningioma \\
\hline 10 & Male/54 & $\begin{array}{l}\text { Right lateral ventricles triangle, deep lobulation, few cystic } \\
\text { areas, mild inhomogeneous signal, moderate edema, a } \\
\text { little bleeding }\end{array}$ & $51 \times 43 \times 51$ & $\begin{array}{l}\text { Identify meningioma } \\
\text { and papilloma } \\
\text { choroideum }\end{array}$ \\
\hline 11 & Female/71 & $\begin{array}{l}\text { Right temporal region, moderate edema, low signal on } \\
\text { T2-weighted imaging, inhomogeneous signal, shallow lobe, } \\
\text { less cystic regions, moderate edema }\end{array}$ & $48 \times 55 \times 53$ & Meningioma \\
\hline 12 & Female/39 & $\begin{array}{l}\text { Left frontal bottom, a little partial cystic region, shallow } \\
\text { lobe, severe edema, close to the falx, low signal on } \\
\text { T2-weighted imaging }\end{array}$ & $35 \times 40 \times 33$ & Meningioma \\
\hline
\end{tabular}

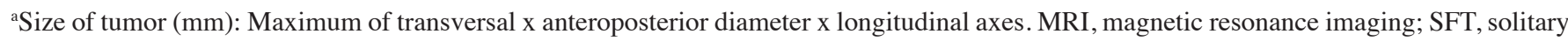
fibrous tumor.

Methods and parameters. A 1.5T superconducting twin-speed magnetic resonance scanner (SignaHDxt 1.5T; GE Healthcare, Chicago, IL, USA) was used for MR examination.

The patients were scanned head first in the supine position. Imaging assessments included plain scanning (without an injection of any contrast medium) and enhancement scanning (injection with a contrast medium). There were two sequences on the plain scan: Axial plane T1-weighted imaging (T1WI) and T2WI. There were three sequences on the enhanced scan: Axial plane fat-suppression (FS)-T1WI, coronal plane FS-T1WI and sagittal plane FS-T1WI.

For the axial plane on the plain scan, the time of repetition/time of echo (TR/TE) was 500/20 msec in T1WI and 4,000/102 msec in T2WI. The enhanced scanning parameters were the same as that of plain scan in the axial plane, while FS technology was used. In the coronal plane FS-T1WI, the TR/TE was
400-500/20-24 msec. The TR/TE was 400-500/20-24 msec for the sagittal plane FS-T1WI. The field of view was $240 \times 240 \mathrm{~mm}$. The layer thickness was $5 \mathrm{~mm}$ and the layer distance was $10 \%$.

The scan was commenced once the patient had been injected with the contrast medium. The contrast medium was a gadopentetatedimeglumine intravenous injection for adults and children aged over 2 , with $0.2 \mathrm{ml} / \mathrm{kg}$ body weight (or $0.1 \mathrm{mmol} / \mathrm{kg}$ ).

Evaluation. Preoperative diagnosis and postoperative evaluation of the MRI were performed by 2 to 3 radiologists with the title of Associate Chief Physician and above. This was initially performed in a blinded manner, then the results of the cases were discussed and a consensus was reached.

Evaluation of tumor peripheral edema. The peripheral edema around the tumor was measured in the MR images, with 

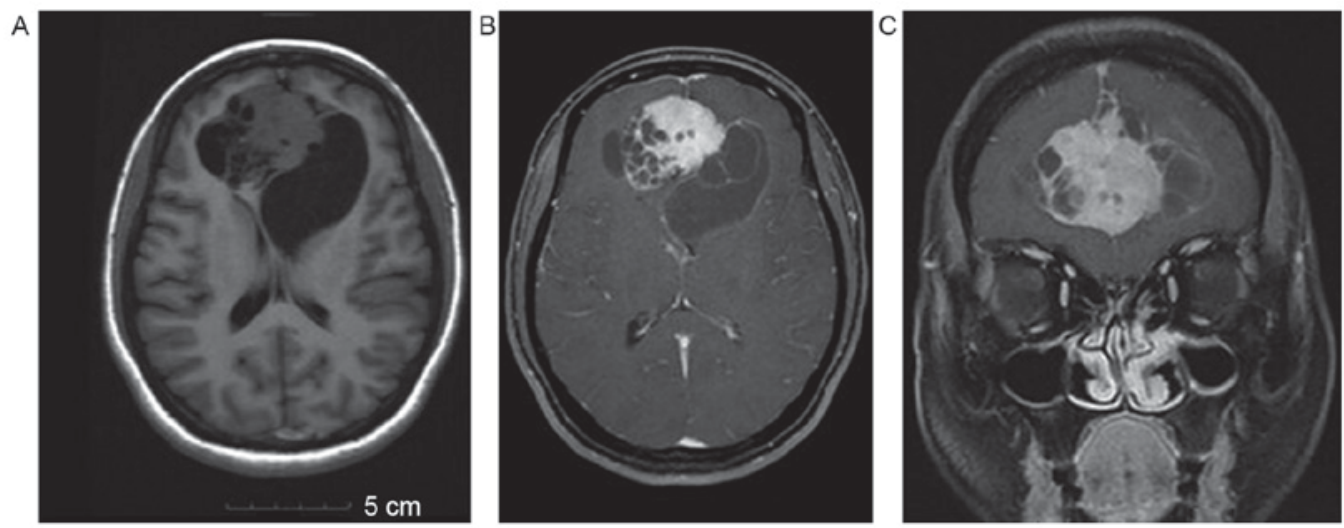

Figure 1. Case 1. One of the features of a solitary fibrous tumor: An inhomegeneneous signal bilateral soft-tissue mass at the falx. (A) Plain scan: TRA-T1WI. (B) Enhancement scan: TRA FS-T1WI. (C) Enhancement scan: COR FS-T1WI. TRA, transverse section; COR, coronal plane; FS, fat suppression; WI, weighted imaging.
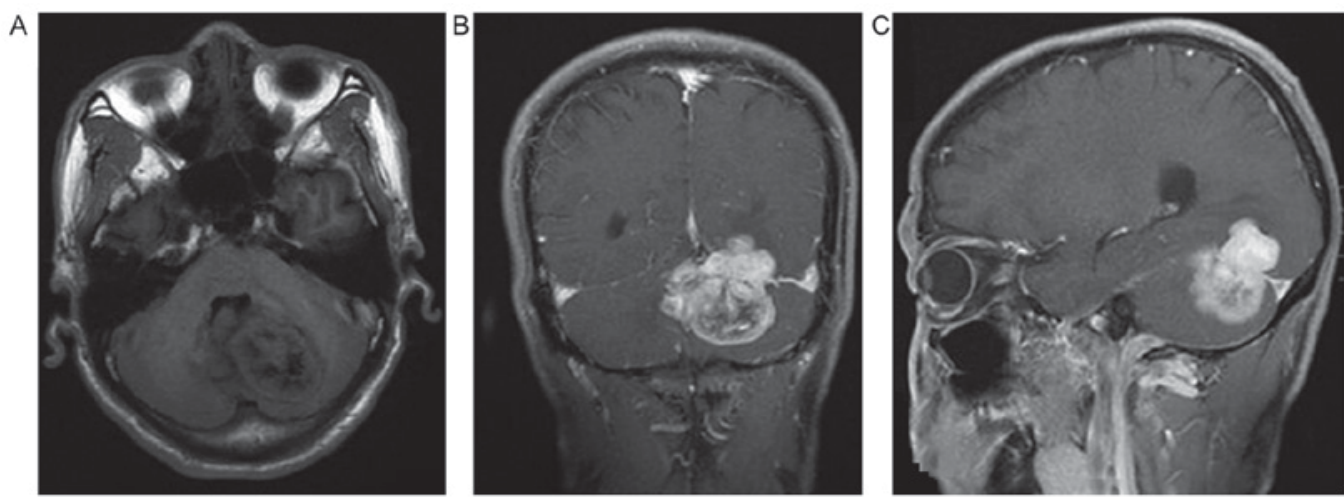

Figure 2. Case 2. One of the features of a solitary fibrous tumor: The mass spanned the tentorium cerebelli. (A) Plain scan: The lesion signal was inhomogeneous; a low to intermediate mixed signal intensity was present on T1WI. (B) Enhancement scan: COR T1WI. (C) Enhancement scan: SAG T1WI. SAG, Sagittal plane; WI, weighted imaging.

an evident edema found around the lesion. The image was divided into four degrees according to the maximum width of the peripheral edema as follows: i) No edema; ii) mild edema, the maximum width of the peripheral edema was $\leq 2 \mathrm{~cm}$; iii) moderate edema, the maximum width of the peripheral edema was $>2 \mathrm{~cm}$ and $\leq 4 \mathrm{~cm}$; and iv) severe edema, the maximum width of the peripheral edema $>4 \mathrm{~cm}$.

Assessment of the lobulated margin of focus. According to the degree of protrusion at the edge of the lesion, the lobulated margin was divided into two types as follows: i) Deep lobulation, the arc chord distance/chord length ratio was $\geq 0.4$; and ii) shallow lobulation, the arc chord distance/chord length ratio was $<0.4$.

\section{Results}

MRI preoperative diagnosis. A total of 12 cases were assessed in the present study, of which, 9 cases were originally diagnosed as meningioma preoperatively. Among these, 5 cases were suggested to possibly be malignant meningioma and 4 cases were benign. A single case was originally believed to have a differential diagnosis of a meningioma and papilloma of the choroid. Another case was originally diagnosed as a glioblastoma, and in only 1 case was a meningioma distinguished as an SFT. For the postoperative diagnosis, all 12 cases were meningeal SFTs. Of the cases, 3 were malignant, 7 were benign and 2 were a borderline tumor.

Growth pattern of the tumor. As observed from MRI, 3 cases spanned the tentorium cerebelli and 3 cases spanned the falx (Figs. 1A-C and 2A-C). A total of 4 superficial tumors were accompanied by adjacent bony destruction, but did not span the tentorium cerebelli or falx. A single case was close to the falx, and another case was located at the triangular area created by the right lateral ventricles, without bone destruction and not crossing the falx or tentorium. All 12 cases invaded and involved a wide range of tissues. The margins of the lesions were irregular and 11 cases had distinct interfaces with the surrounding structures; only 1 case did not.

Shape and lobulation. Observed from MRI, the shapes of the 12 cases were irregular. The margins of all the cases were not smooth, with 7 cases of deep lobulation and 5 cases of shallow lobulation.

MRI signal change. In the plain scans, the lesions of the 12 cases usually exhibited inhomogeneous signals. The tumors were of low to intermediate mixed signal intensity on the T1WI, and 3 had a medium to slightly higher signal 
(which may be associated with a small quantity of bleeding). A substantial region of the tumor was of medium low or high signal on the T2WI (Fig. 3A and B). An irregular low signal intensity was observed in the lesions of 7 cases. Combined with the T1WI and T2WI plain scans, the outer limits of the substantial region of the tumor also showed larger cystic areas in 5 cases. Cystic vacuole lesions were modified in the cystic areas in 2 cases, a marked 'capsule' change was observed in the 2 cases. In the enhanced scans, a substantial region of the tumor was markedly enhanced (Figs. 1A-C and 2A-C). There was no enhancement in the cystic region. The cyst wall was circularly enhanced. The signal of the tumor was extremely uneven in the 12 cases of the group. Non-enhanced zones, largely in the tumors, shown as multiple small strips, where patchy tracts could be found. The signal in the focus became patchy and inhomogeneous in 10 cases. Non-enhanced zones in the focus were shown as layered, with low signal, in 2 cases.

Tumor hemorrhage. Tumor hemorrhage occurred in only 3 cases, and could be observed as a slightly higher signal on the plain T1WI scan. There was no bleeding in the remaining 9 cases.

Tumor peripheral edema. Observed from MRI, there was mostly no or mild to moderate edema around the lesion. No edema was present around the lesions in 3 cases, while 4 cases presented with mild edema and 4 cases with moderate edema around the lesions. Only 1 case exhibited severe edema around the lesion.

\section{Representative cases}

Case 1. In the representative case of a 40-year-old woman shown in Fig. 1, the MR plain scan showed an inhomogeneous signal bilateral soft-tissue mass of the falx. The tumor spanned the falx, with deep lobulation, a polycystic variable region and a clear edge. There was no edema around the lesion, and the mass showed inhomogeneous enhancement (Fig. 1).

Case 2. In the representative case of a 53-year-old man shown in Fig. 2, MR enhancement showed that there was an inhomogeneous signal soft-tissue mass spanning the tentorium cerebelli. The tumor had deep lobulation, partial cystic areas and a clear edge. There was mild peripheral edema around the mass. A substantial region of the tumor was markedly enhanced. Inhomogeneous enhancement was also present (Fig. 2).

Case 3. In the representative case of a 52-year-old woman shown in Fig. 3, the lesion was located at the left occipital lobe and left cerebellum. The tumor spanned the tentorium cerebelli and presented with an inhomogeneous signal. The mass exhibited an uneven low signal on T2WI. An irregular low signal intensity was observed in the lesion (Fig. 3).

Change in adjacent tissues. The lesions were close to the dura mater in 11 cases. The adjacent dura had no typical characteristics of the 'dural tail sign'. Only 1 case was at the triangular area created by the right lateral ventricles. There was no clear association with the meninges in the MR images.

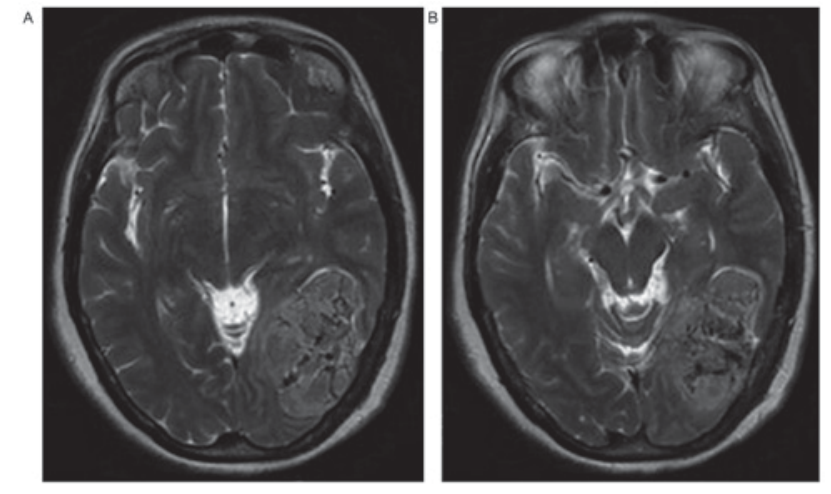

Figure 3. Case 3. A patchier low signal intensity was observed in the lesion. (A and B) Plain scan: T2WI.

\section{Discussion}

The SFT has gradually been recognized as an independent tumor since it was first reported in 1931. The tumor was once believed to occur only in the pleura and to originate from the tissue of the mesothelium (15). Later, it was revealed that an SFT could occur almost anywhere in the body, including the dura, orbital cavity, lungs, liver, kidneys and particularly the pleura. Approximately $80 \%$ of SFTs originate in the pleural space $(2,13)$. Another previous study suggested that $60 \%$ were located outside of the pleura (16), as SFTs have occurred on the falxcerebri, the convexity of the brain, the saddle area, the posterior cranial fossa, the dura mater, the encephala and in the CNS. SFTs often originate in the dura mater (17) and have been reported to be ubiquitous neoplasms of mesenchymal origin (18). SFT originating from the meninges are rare overall; the world literature on solitary fibrous tumors of the central nervous system (CNS) between August 1996 and July 2011 were reviewed, only 220 cases were reported in CNS, and 170 cases of SFT were originated from the meninges (19) ISFT is rare, accounting for $\sim 0.6 \%$ of all intracranial tumors $(13,20,21)$. Reports of meningeal SFTs have gradually increased, however, an understanding of its image characteristics is lacking. In the present study, the imaging characteristics of 12 cases were discussed in order to assist the recognition and understanding of this disease.

With regard to sex and age, the ages of the patients at the diagnosis of SFT range greatly, for example, being reported as between 5 and 87 years in one study (22). Another literature review reported a median age of 44.5-55.0 years (23). The ages of other reported cases have been within this range (24-27). In the present study, the ages of the patients ranged between 20 and 71 years, with a mean age of 48.8 years. The results from this group were similar to those from the literature review. Viewpoints on the incidence in males and females were highly varied. Certain studies identified no gender differences $(13,21,28)$. However, another study (29) deemed that the proportion of male patients with SFT was slightly higher than that of female patients. Meningiomas were also reported to be more common in middle-aged women. Other reports showed that the male to female ratio of cases was 3:1, 4:14 and 23:20 (30-32). There were 12 cases in the present study, with 6 men and 6 women, giving a 1:1 ratio and no difference in sex predominance. The research results in the present study 
were similar to that of certain aforementioned studies and the study by Nawashiro et al (29), but the number of patients in these studies is small, and further observations are therefore required to confirm the ratio between men and women.

The shape of an SFT has been reported as round, oval or irregular (14). The result in the present group is similar to that reported previously (13); that is, there were 4 oval cases and 8 irregular cases in the present group. All showed different degrees of lobulation, namely, 7 cases presented with deep lobulation and 5 cases with shallow lobulation. With regard to the size, one previous study (29) reported that the tumor size was not the same in intracranial-SFT (I-SFT). The smallest reported diameter was $\sim 2 \mathrm{~cm}$ and the largest was $6 \mathrm{~cm}$. The sizes of the lesions differed greatly within the present study. The majority of the lesions were large, with the largest diameter at $>7 \mathrm{~cm}$, and the smallest was $>3 \mathrm{~cm}$ in diameter. In addition, the volume of the lesions was larger in all cases and the span of the size of the lesions was also larger. However, the results of the present study were similar to those of previous reports.

Meningeal SFT originates in the dura materand shares characteristics of intracranial-extracerebral tumors. Therefore, observations of the boundary between the tumor and surrounding brain tissue show that the boundary of the lesion is clear (21). The result of the present study was similar to these findings. The boundaries in 11 cases were clear, with an unclear boundary in 1 case only.

The pathological characteristics of the tumor tissue in an SFT under the microscope show tumor cells that are spindled, and cell arrangement that is sparse and densely interphase (23). Different proportions of collagenous fibers are present. The pathological characteristics of the tumor are similar to that of a fibrous-type (fibroblastic) meningioma. SFT is often clinically misdiagnosed as a meningioma or another extra-axial tumor. Certain studies have, however, indicated an SFT in the CNS at the preoperative diagnosis. The correct rate of diagnosis of an SFT has been reported as almost 0 in other areas of the pleura $(13,14,21)$. The present result was similar to this; 11 cases were not considered to be an SFT based on the original preoperative MRI. Only 1 case was considered to have a differential diagnosis of meningioma and SFT, however, it was also originally misdiagnosed as a meningioma. The preoperative diagnosis from MRI in the present study was consistent with that reported in the literature. As there are a number of similarities between the features of an SFT and that of certain other tumors (particularly fibrous-type meningioma) with regard to image changes and pathological features, the SFTs were easily misdiagnosed upon imaging. Moreover, there was no specificity of clinical symptoms and the tumors were mostly associated with the dura mater. Therefore, it was often misdiagnosed as meningioma.

The T1WI features of a SFT in the CNS and meningioma have been considered to be similar (22,31-33), with the SFT displaying a slightly lower T1WI signal and a high or mixed T2WI signal. The MR findings of I-SFT were described in the study by Wang et al (24). It was reported that I-SFT was usually a solid lesion with clear edges, which exhibited a medium T1WI signal, a low T2WI signal and was significantly enhanced upon contrast-enhanced scanning. Nawashiro et al (29) suggested that SFT was characterized by high and low hybrid signals in the T2 sequence, which is known as the 'black and white sign'.
Clarençon et al (34) described the specific performance of intracranial SFTs as a 'yin-yang sign', as there were diametric boundaries between the low signal area and a slightly high signal area. When enhanced, the low signal area on plain scan was markedly enhanced.

Overall, the results were similar in the present group, but there were a few differences. In the 12 cases in the study, the signal was mainly a low or medium mixed T1WI signal. There were a few patchy uneven medium and slightly higher signals in 3 cases that may have been associated with a small quantity of bleeding. When observing the cases using T2WI, the solid region of the tumors showed high and low mixed signals. Among those, the lesions in 7 cases could be clearly observed with a cord-like or patchy low signal, referred to previously as the 'black and white sign' or the 'yin-yang sign' (21). Further detail was also observed in the present study and this may be associated with the different collagen fiber content of the tumor. The cystic lesion area presented with a low T1WI signal and a high T2WI signal.

Signal changes of the tumor in the present study were consistent with previous reports $(22,29,33)$. However, the results were slightly different from the 'usually solid lesions' described in the study by Wang et al (24). There were large cystic regions in the certain tumors of the present study (5/12 cases, $41.67 \%)$, and there were small cystic areas in others (3/12 cases, $25.00 \%$ ). Moreover, no fluid-attenuation fluid inversion recovery (FLAIR) sequence imaging was performed in the present study, so the characteristics of an SFT using FLAIR could not be compared. This will require further studies, in which a greater number of cases should be compared and analyzed.

Another MR sign is cystic degeneration and necrosis in the lesion. There were large cystic areas in the tumor in 5 cases; a marked 'capsule' change was observed in 2 of the cases. The result was the same as that in a previous study (2). The fact that there are large cystic areas or 'capsule' changes in the tumor could be a potential important feature of an SFT upon MRI.

Spindled fibroblast-like cells have been reported in the SFT pathology (24). The tumors contain a significant amount of collagen fiber and scar tissue. The cell sparse zone and dense region is arranged in phases, and is hypervascular. Large amounts of collagen fibers are present in the cell sparse zone, so that a low T2WI signal is evident. Less slender collagen fibers are apparent in the cell dense region, so a moderate or slightly high T2WI signal is evident (2). Nawashiro et al (29) also analyzed the signal of the collagen fibers in the tumor and found that they showed a low signal on the T2 sequence and a high signal in the area of blood vessels. A characteristic feature was a high and low mixed T2WI signal, referred to as the 'black and white sign' in the aforementioned text. This sign was often apparent in an SFT, but less so in meningioma, and may be another important feature that may contribute to the diagnosis of an SFT upon MRI.

The different contents of the tumor tissue influence the signal changes found by MRI. Different contents of different tissues lead to the different signal features in the images. Therefore, the signal changes of an SFT are associated with the different contents of different tissues $(2,14)$. In the present study, an abundant number of spindle cells was found upon pathological examination. The tumor cells were 
spindle-shaped or oval. SFT cells were small and present in spiral, woven and radial arrangements. The cells were sparsely and densely arranged in alternate phases. Proliferation of collagen fibers was found in the tumor cells. Certain cases exhibited degeneration, including cystic changes in the tumor. Mucus changes were also observed focally. The T1WI and T2WI signal features of the cases were consistent with the pathological characteristics, so inhomogeneous signals in an SFT were often observed.

Due to an abundance of collagen fibers rich in blood vessels, and the different contents of different tissues, the degree of enhancement of the lesions varied with the contents of the types of tissues. The degree of tumor enhancement was different in the report literature (33), with mild, moderate and significant uniform or uneven enhancement. The degree of tumor enhancement was reported to be associated with the collagen fibers, matrix and blood vessel content in the study by Chong et al (35). As SFTs are tumors with a rich blood supply, with a different organizational structure, they were often noted as heterogeneous. Significantly tumor enhancement was noted in the cell dense regions and blood vessel-rich regions. However, enhancement was not evident in cell sparse regions and areas lacking vascular structure (2), similar to the results in the present study. The essence of the tumor (the cell dense region and blood vessel-rich region) was markedly enhanced in the present study. This phenomenon suggested that the SFT was rich in vascularity and in accordance with the pathological findings. Moreover, the signal in the tumor was uneven. The non-enhancement zone was visible in the shape of a cord-like patch. The majority of the lesions were significantly uneven in the enhanced scanning. Now, the changes in the tumor were non-uniform. For certain tumors, the low signal areas exhibited layered signal intensity. The non-uniform distribution change was visible in 10 cases. The layered non-enhancement low signal was found in the lesions of 2 cases in the cell sparse region. These results were also consistent with the pathological characteristics. The cystic areas were non-enhanced, but the cyst wall showed a moderate circular enhancement. The difference in enhancement in the cystic region and cystic wallof the SFT also hinted at the diversity of the histological compositions and enhancement patterns.

Dynamic enhancement was observed in a previous study (36), but the dynamically enhanced features of the SFT were influenced by a number of factors. Due to the SFT being a tumor containing a rich blood supply with a large number of collagen tissues and all types of modified ingredients, its proportion of cells and collagen was different. Therefore, the dynamically enhanced features of the SFT were influenced by a number of factors. When the dynamic enhancement of the arterial phase to the delayed phase was observed in certain reports, the essence of the tumor showed a continuous filling type (13). Another study (34) reported that the low T2WI signal in the tumor with significantly progressive enhancement was associated with the density of the collagen fibers. The feature was a hint that the tumor would later be diagnosed as an SFT. Due to the lack of experience, only routine enhanced imaging was performed in the preoperative imaging of the present study, and dynamic enhancement was not performed, so these features could not be observed. This therefore requires further research.
The literature does not include much discussion on the growth mode of an SFT. MRI findings of an I-SFT were observed in a study by Dai et al (28), which found that the lesions crossed the tentorium cerebelli in 2 cases (2/8 cases), similar to the present study in which it crossed the tentorium cerebelli in 3 cases (3/12 cases). By observing the growth method of an SFT on MRI, it was found that the tumor was often spanning growth $(6 / 12$ cases, $50 \%)$, meaning thatthe lesion spanned both sides of the tentorium cerebella or the cerebral falx; 3 cases spanned the tentorium cerebelli and 3 cases spanned the cerebral falx. Wide ranges were involved.

Literature information is lacking on the changes to the adjacent skull bones, and the results presented vary. One study (37) indicated that the adjacent bone, nerve and brain parenchyma near the tumor were involved when 51 cases of CNS SFTs from the literature were reviewed. Other studies $(38,39)$ reported structural changes of the tumor of the orbiton computed tomography (CT) scans. However, compression bone remodeling was rare and without bony erosion in the imaging. If bone destruction was observed clearly on CT, it would reflect the malignant biological behavior of the tumor. However, other studies $(38,39)$ showed that the adjacent bone was evidently damaged or corroded in the orbital SFT. The present results were roughly consistent with the aforementioned studies in regard to identification of bone structure alterations and done destruction, 4 cases (4/12 cases) with adjacent bony destruction were detected. The lesions in these 4 cases were located on the convexity of the brain and were superficial tumors. Among the 4 cases, there was 1 case spanning the falx with adjacent bony destruction. The other 3 cases did not span the tentorium or falx, but invaded the adjacent skull. All the lesions in this group had a large size and a wide range of tissues involved. Although the boundary between the lesion and surrounding tissue was clear, the edge of the lesion was mostly not neat.

Nawashiro et al (29) studied the changes in the adjacent meninges. The study suggested that the SFT was attached to the dura mater, but the 'dural tail sign' was rarely observed. It was suggested that the formation mechanism of the SFT was different from that of meningioma, but other studies presented different views $(21,29)$ : It was discussed that the enhancement was markedly inhomogeneous and the 'dural tail sign' may occasionally be visible. In the present study, the tumor was attached to the dura mater, but there was no typical 'dural tail sign'. This result was similar to that found by Nawashiro et al (29).

I-SFT is often derived from the meninges; it has characteristics of extra cerebral tumors, and is coated by a fibrous capsule. Edema in the peripheral brain parenchyma is rare (21). In the present study, mild or no edema could be commonly found. The cases were as follows: 3 cases without edema and 4 cases with mild edema. Moderate edema was observed in 4 cases and only 1 case presented with severe edema. This last case may have been associated with the size of the lesion and oppression of the adjacent vein or sinus. This situation was consistent with the experimental results reported in other studies (40).

The occurrence rate of an SFT is low, and clinically, doctors are unfamiliar with the tumor (28). The characteristics of the tumor are similar to other tumors upon imaging. Moreover, the tumor has no specificity in clinical symptoms, so it is often misdiagnosed as other tumors (28). While SFT 
should be differentiated from other tumors occurring in the meninges, the diagnosis may easily be confused as an SFT is morphologically similar to other tumors, such as meningioma, particularly fibrous (fibroblastic) meningioma, angiomatous-type meningioma, hemanyiopericytoma, neurilemmoma and hemangiopericytoma (28). The differential diagnosis requires further research. Although the signal changes could reflect more pathological changes in the lesions, the final diagnosis and differential diagnosis must be confirmed by pathological and immunohistochemical analysis (41).

In summary, intracranial SFTs often occur in or close to the meninges. The MRI features of a meningeal SFT are similar to other tumors in the CNS, such as fibrous meningioma. However, certain individual characteristics are also present. The present study and literature review indicated that meningeal SFTs were often solitary, large, soft-tissue masses with an irregular edge and a clear boundary, with a lobulated contour and involving a wide range of tissues. The tumor often grew spanning the falx or the tentorium cerebelli. A significantly inhomogeneous signal was often found upon MRI, particularly where the T2WI signal was low or low-high mixed. This characteristic is often called the 'black and white sign' or 'yin-yang sign'. The majority of the lesions were significantly uneven in the enhanced scanning. The changes in the tumor were map-like ('map sign'). For certain tumors, the low signal areas looked like a 'chrysanthemum' (called 'chrysanthemum sign'), lacking the typical 'dural tail sign'. There was a large cystic area in the tumor, particularly in the periphery of the lesion, with little or no bleeding. The aforementioned features are useful for the identification of SFTs and other tumors, such as fibrous meningioma.

In addition, only MR scans were performed, not CT scans, in the present study. No dynamically enhanced scan was performed and the characteristics of changes in gradual enhancement in dynamic scanning requires additional investigation. Moreover, the number of cases in this study was small and therefore insufficient imaging characteristics may have been studied. In future studies, the characteristics of an SFT in MRI require further analysis.

\section{References}

1. Alawi F, Stratton D and Freedman PD: Solitary fibrous tumor of the oral soft tissues: A clinicopathologic and immunohistochemical study of 16 cases. Am J Sur Pathol 25: 900-910, 2001.

2. Wagner E: Das tuberkelahnliche lymphadenom (der cytogene oder reticulirte tuberkel). Arch Heilk (Leipzig) 11: 497, 1870 (In German).

3. Klemperer P and Coleman BR: Primary neoplasms of the pleura: A report of five cases. Am J Ind Med 22: 1-31, 1992.

4. de Perrot M, Fischer S, Bründler MA, Sekine Y and Keshavjee S: Solitary fibrous tumors of the pleura. Ann Thorac Surg 74 285-293, 2002

5. Guinee DG and Allen TC: Primary pleural neoplasia: Entities other than diffuse malignant mesothelioma. Arch Pathol Lab Med 132: 1149-1170, 2008.

6. Zhang ZL, Gu XW, Xiao Q and Wang CM: Clinicopathological analysis of solitary fibrous tumor in 11 patients. Cancer Res Clin 27: 107-108,112, 2015 (In Chinese).

7. Mekni A, Kourda J, Hammouda KB, Tangour M, Kchir N, Zitouna M and Haouet S: Solitary fibrous tumour of the central nervous system: Pathological study of eight cases and review of the literature. Pathology 41: 649-654, 2009.

8. Carneiro SS, Scheithauer BW, Nascimento AG, Hirose T and Davis DH: Solitary fibrous tumour of the meninges: A lesion distinct from fibrous meningioma. A clinicopathologic and immunohistochemical study. AM J Clin Pathol 106: 217-224, 1996.
9. Lococo F, Cesario A, Cardillo G, Filosso P, Galetta D, Carbone L, Oliaro A, Spaggiari L, Cusumano G, Margaritora S, et al: Malignant solitary fibrous tumors of the pleura: Retrospective review of a multicenter series. J Thorac Oncol 7: 1698-1706, 2012.

10. Val-Bernal JF, Mayorga M, Fernández F, Parra A, Crespo J and García-Polavieja M: Solitary fibrous tumors arising from the mesentery of adult patients. Report of two cases and review of the literature. Rom J Morphol Embryol 55: 203-207, 2014.

11. Bisceglia M, Dimitri L, Giannatempo G, Carotenuto V, Bianco M, Monte V, D'Angelo V and Magro G: Solitary fibrous tumor of the central nervous system: Report of an additional 5 cases with comprehensive literature review. Int J Surg Pathol 19: 476-486, 2011.

12. Yan J, Ahl KL, Manning KA, Mann FA and Lewis DH: Radiology-pathology conference: 18F-FDG PET-CT imaging of solitary fibrous tumor of the pleura. Clin Imaging 37: 598-601, 2013.

13. Cui J, Han LX, Cao HX, Du WQ, Zhang L, Wang J and Chen G: MRI characteristic of intracranial solitary fibrous tumors. Radiol Pract 31: 224-227, 2016 (In Chinese).

14. Liu H, Yang ZQ, Wang YT, Liu X, Chen H, Zhang TJ and Luo KJ: CT and MRI findings of intracranial solitary fibrous tumor. J Clin Radiol 30: 1265-1268, 2011 (In Chinese).

15. Chen H, Zeng XW, Wu JS, Dou YF, Wang Y, Zhong P, Xu R, Jiang CC and Wang XQ: Solitary fibrous tumor of the central nervous system: A clinicopathologic study of 24 cases. Acta Neurochir (Wien) 154: 237-248, 2012.

16. Zhanlong M, Haibin S, Xiangshan F, Jiacheng S and Yicheng N: Variable solitary fibrous tumor locations: CT and MR imaging features. Medicine (Baltimore) 95: e3031, 2016.

17. Smith AB, Horkanyne-Szakaly I, Schroeder JW and Rushing EJ: From the radiologic pathology archives: Mass lesions of the dura: Beyond meningioma-radiologic-pathologic correlation. Radiographics 34: 295-312, 2014.

18. Savastano S, d'Amore ES, Beghetto M, Borgo DD, Franceschetti I and Capalbo M: A presacral solitary fibrous tumor with extramedullary hematopoiesis: Radiologic and pathologic findings. Rare Tumors 5: e61, 2013

19. Bisceglia M, Galliani C, Giannatempo G, Lauriola W, Bianco M D'angelo V, Pizzolitto S, Vita G, Pasquinelli G, Magro G and Dor DB: Solitary fibrous tumor of the central nervous system: A 15-year literature survey of 220 cases (August 1996-July 2011). Adv Anat Pathol 18: 356-392, 2011.

20. Tihan T Viglione M, Rosenblum MK, Olivi A and Burger PC: Solitary fibrous tumors in the central nervous system. A clinicopathologic review of 18 cases and comparison to meningeal hemangiopericytomas. Arch Pathol Lab Med 127: 432-439, 2003.

21. Ke DB, Liu WK, Zhang S, Yu Y, Zhang YK and Hui XH: MRI manifestations of intracranial solitary fibrous tumor. West China Med J 32: 46-50, 2017 (In Chinese).

22. Fargen KM, Opalach KJ, Wakefield D, Jacob RP, Yachnis AT and Lister JR: The central nervous system solitary fibrous tumour: A review of clinical, imaging and pathologic findings among all reported cases from 1996 to 2010. Clin Neurol Neurosurg 113: 703-710, 2011.

23. Zhang X, Wang H, Wang S, Miao J, Piao Z and Dong Y: Clinicopathological analysis of solitary fibrous tumor. ChineseGerman J Clin Oncol 11: 282-284, 2012.

24. Wang C, Manucha V, Faro S, Weaver M and Mukherjee AL: Fourth ventricular solitary fibrous tumor: A case report and review of the literature. J Med Case Rep 6: 205, 2012.

25. Robert T, Duc C, San Millán Ruíz D and Morard M: Solitary fibrous tumour with intramedullary component: Case report and review of the literature. Neurol Neurochir Pol 48: 144-149, 2014.

26. Treglia G, Oragano L, Fadda G, Raffaelli M, Lombardi CP, Castaldi $\mathrm{P}$ and Rufini V: A rare case of solitary fibrous tumor of the adrenal gland detected by (18)F-FDG PET/CT. Clin Nucl Med 39: 475-477, 2014.

27. Dong A, Zuo C, Wang Y and Cui Y: Enhanced CT and FDG PET/CT in malignant solitary fibrous tumor of the lung. Clin Nucl Med 39: 488-491, 2014.

28. Dai Y, Long LL and Ye W: MR imaging features of intracranial solitary fibrous tumors. Radiol Pract 30: 127-130, 2015 (In Chinese).

29. Nawashiro H, Nagakawa S, Osada H, Katoh H, Ohnuki A, Tsuzuki N, Miyazawa T, Shima K, Ogata S and Aida S: Solitary fibrous tumor of the meninges in the posterior cranial fossa: Magnetic resonance imaging and histological correlation-case report. Neurol Med Chir (Tokyo) 40: 432-434, 2000.

30. Caroli E, Salvati M, Orlando ER, Lenzi J, Santoro A and Giangaspero F: Solitary fibrous tumors of the meninges: Report of four cases and literature review. Neurosrug Rev 27: 246-251, 2004. 
31. Tihan T, Viglione M, Rosenblum MK, Olivi A and Burger PC Solitary fibrous tumors in the central nervous system. A clinicopathologic review of 18 cases and comparison to meningeal hemangiopericytomas. Arch pathol Lab Med 127: 432-439, 2003.

32. Metellus P, Bouvier C, Guyotat J, Fuentes S, Jouvet A, Vasiljevic A, Giorgi R, Dufour H, Grisoli F and Figarella-Branger D: Solitary fibrous tumors of the central nervous system: Clinicopathological and therapeutic considerations of 18 cases. Neurosurgery 60 715-722, 2007.

33. Wang XQ, Zhou Q, Li ST, Liao CL, Zhang H and Zhang BY: Solitary fibrous tumors of the central nervous system: Clinical features and imaging findings in 22 patients. J Comput Assist Tomogr 37: 658-665, 2013

34. Clarençon F, Bonneville F, Rousseau A, Galanaud D, Kujas M, Naggara O, Cornu P and Chiras J: Intracranial solitary fibrous tumor: Imaging findings. Eur J Radiol 82: 387-394, 2011.

35. Chong S, Kim TS, Cho EY, Kim J and Kim H: Benign localized fibrous tumour of the pleura: CT features with histopathological correlations. Clin Radiol 61: 875-882, 2006.
36. Zhang WD, Chen JY, Cao Y, Liu QY and Luo RG: Computed tomography and magnetic resounance imaging findings of solitary fibrous tumors in the pelvis: Correlation with histopathological findings. Eur J Radiol 78: 65-70, 2011.

37. Yang BT, Song ZL, Wang YZ, Dong JY and Wang ZC: Solitary fibrous tumor of the sinonasal cavity: CT and MR imaging findings. AJNR Am J Neuroradiol 34: 1248-1251, 2013.

38. Glazer-Hockstein C, Syed NA, Warhol M and Gausas RE: Malignant solitary fibrous tumor metastatic to the orbit. Ophthal Plast Reconstr Surg 20: 471-473, 2004.

39. Woo KI, Suh YL and Kim YD: Solitary fibrous tumor of the lacrimal sac. Ophthal Plast Reconstr Surg 15: 450-453, 1999.

40. Han YP, Zhang YT, Liu JL, Zhang XL and Zhou JL: Comparison of imaging and pathological findings of intracranial hemangiopericytoma and solitary fibrous tumor. Chin J Magn Reson Imaging 6: 917-924, 2015 (In Chinese).

41. Sun J, Yu XR, Shi BB, Zheng J and Wu JT: CT features of retroperitoneal solitary fibrous tumor: Report of three cases and review of the literature. World J Surg Oncol 12: 324, 2014. 